\title{
Heat Capacity Studies of $\mathrm{NdNi}_{4} \mathrm{Si}$ Compound
}

\author{
M. Falkowski ${ }^{a, *}$, M. Reiffers ${ }^{b}$, M. Zapotoková $^{b}$, A. KowalczyK $^{a}$, T. Toliński $^{a}$ \\ AND E. GAŽO ${ }^{b}$ \\ ${ }^{a}$ Institute of Molecular Physics, Polish Academy of Sciences \\ M. Smoluchowskiego 17, 60-179 Poznań, Poland \\ ${ }^{b}$ Institute of Experimental Physics, Slovak Academy of Sciences \\ Watsonova 47, 04353 Košice, Slovakia
}

\begin{abstract}
The study of the heat capacity of the intermetallic compound $\mathrm{NdNi}_{4} \mathrm{Si}$ including the effect of the magnetic field is reported. This compound crystallizes in the hexagonal $\mathrm{CaCu}_{5}$-type structure, space group $P 6 / \mathrm{mmm}$. $\mathrm{NdNi}_{4} \mathrm{Si}$ is ferromagnetic with $T_{\mathrm{C}}=8 \mathrm{~K}$ and the saturation magnetic moment of $1.5 \mu_{\mathrm{B}} /$ f.u. at $4.2 \mathrm{~K}$ (in $H=9 \mathrm{~T})$. The heat capacity was analyzed considering the electronic contribution, the Schottky anomaly, and the lattice contributions in the frames of the Debye model. The scheme of the energy levels created by the crystal electric field split is determined from the Schottky contribution to the specific heat. $\mathrm{NdNi}_{4} \mathrm{Si}$ was characterized by the electronic heat capacity coefficient $\gamma=85 \mathrm{~mJ} /\left(\mathrm{mol} \mathrm{K}^{2}\right)$ and the Debye temperature $\Theta_{\mathrm{D}}=325 \mathrm{~K}$.
\end{abstract}

PACS numbers: 65.40.De, 65.40.gd, 71.20.Eh, 71.70.--d

\section{Introduction}

Intermetallic compounds of rare earth $(\mathrm{R})$ atoms and transition metal atoms are of great importance both for technological application as well as from the viewpoint of basic research. Apart from La, Ce, Pr and Yb, these compounds order ferromagnetically with $T_{\mathrm{C}}$ in the range from $5.7 \mathrm{~K}(\mathrm{Tm})$ to $22 \mathrm{~K}(\mathrm{Gd})[1]$. The $T_{\mathrm{C}}$ values of $\mathrm{RNi}_{4} \mathrm{Si}$ follow the de Gennes function for heavy rare earths. The reduction observed in the case of light rare earth can be ascribed to the crystal electric field effect. The transition temperatures are smaller compared to their respective parent compounds $\mathrm{RNi}_{5}[2,3]$. In our previous paper [4] we presented results of magnetic measurements for $\mathrm{CeNi}_{4} \mathrm{Si}$. In this paper, we present the magnetic and electronic studies of $\mathrm{NdNi}_{4} \mathrm{Si}$ compound by heat capacity and magnetometric measurements.

\section{Experimental details}

The polycrystalline $\mathrm{NdNi}_{4} \mathrm{Si}$ compound was prepared by the induction melting of stoichiometric amounts of the constituent elements in a water-cooled boat, under an argon atmosphere. The ingot was inverted and melted several times to insure homogeneity. The crystal structure was established by a powder X-ray diffraction technique (XRD), using $\mathrm{Cu} K_{\alpha}$ radiation. $\mathrm{NdNi}_{4} \mathrm{Si}$ compound crystallizes in the hexagonal $\mathrm{CaCu}_{5}$-type structure, space group $P 6 / \mathrm{mmm}$. The room temperature XRD revealed that the $\mathrm{NdNi}_{4} \mathrm{Si}$ compound was single-phase. Magnetic measurements were carried out using the System MagLab2000 magnetometer in a magnetic

\footnotetext{
* corresponding author; e-mail: falkowski@ifmpan.poznan.pl
}

field up to $9 \mathrm{~T}$. Heat capacity measurements were performed by PPMS commercial device (Quantum Design) in the temperature range $2-300 \mathrm{~K}$ and in magnetic field up to $3 \mathrm{~T}$ by the relaxation method using two-tau model.

\section{Results and discussion}

Our magnetic measurements indicate that $\mathrm{NdNi}_{4} \mathrm{Si}$ is ferromagnetic (Fig. 1) with magnetic moment of $1.5 \mu_{\mathrm{B}} /$ f.u. at $4.2 \mathrm{~K}$ and $H=9 \mathrm{~T}$. The magnetic moment value obtained from extrapolation of $M$ to $1 / H=0$ at $4.2 \mathrm{~K}$ is equal to $1.8 \mu_{\mathrm{B}} /$ f.u. In Fig. 1 (inset) the temperature dependences of the magnetization in the zero-field cooling (ZFC) and field cooling (FC) mode show a magnetic phase transition (paramagnetic-ferromagnetic) at temperature $T_{\mathrm{C}}=8 \mathrm{~K}$.

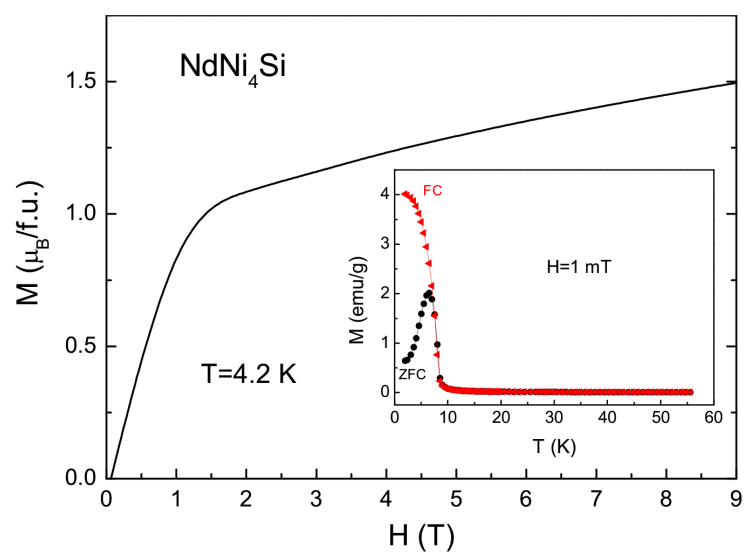

Fig. 1. Magnetization as function of applied magnetic field. Inset: temperature dependence of magnetization for $\mathrm{NdNi}_{4} \mathrm{Si}$ in the $\mathrm{ZFC}$ and $\mathrm{FC}$ mode. 


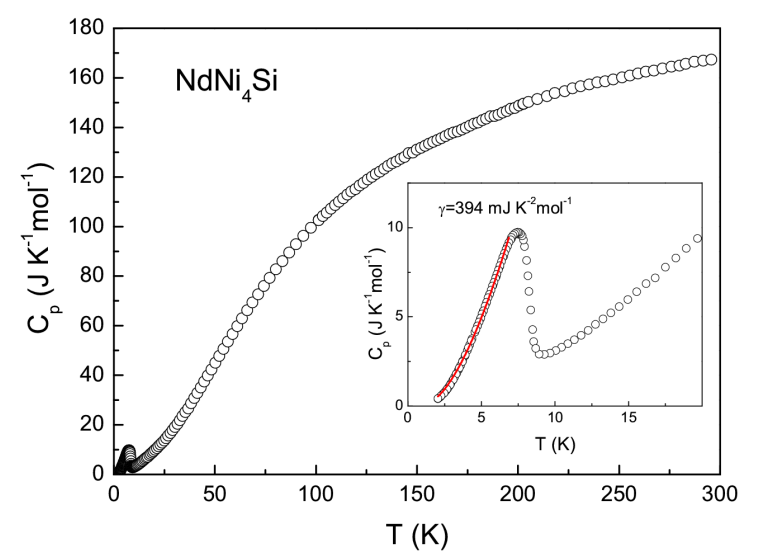

Fig. 2. Temperature dependence of the specific heat of $\mathrm{NdNi}_{4} \mathrm{Si}$. Inset: the low temperature range $2-8 \mathrm{~K}$ is fitted with the formula: $C_{p}(T)=\gamma T+\beta T^{3}+\delta T^{3 / 2}$.

In $\mathrm{RNi}_{4} \mathrm{Si}$ compounds, the magnetic moments of rare earth $\mathrm{R}$ are reduced compared to the free ion values [1]. This occurs probably due to the crystal field interactions. It can be partly ascribed to a random distribution of the easy and hard magnetization axes of the grains composing the polycrystalline sample. However, we have also observed this decreased value of the magnetic moment in neutron diffraction on the $\mathrm{RNi}_{4} \mathrm{Al}(\mathrm{R}=\mathrm{Nd}, \mathrm{Tb}, \mathrm{Dy})$ compounds $[5,6]$.

The total specific heat consists of electronic, phonon and magnetic contributions. The electronic coefficient provides information concerning the conduction band density of states at the Fermi level. Figure 2 shows the temperature dependence of the specific heat for $\mathrm{NdNi}_{4} \mathrm{Si}$ compound. The sharp peak with a maximum at $8 \mathrm{~K}$ in a zero magnetic field, is ascribed to the transition into a magnetically ordered phase. This value is in good agreement with dc susceptibility measurements.

The temperature dependence of the specific heat for $\mathrm{NdNi}_{4} \mathrm{Si}$, can be described by the standard formula

$$
\begin{gathered}
C_{p}(T)=\gamma T+\frac{9 N R}{1-\alpha_{\mathrm{D}} T}\left(\frac{T}{\Theta_{\mathrm{D}}}\right)^{3} \\
\times \int_{0}^{\Theta_{\mathrm{D}} / T} \frac{x^{4} \exp (x) \mathrm{d} x}{[\exp (x)-1]^{2}},
\end{gathered}
$$

where the first and second term correspond to the electronic and phonon contribution, respectively. $N=6$ is the number of the atoms in the formula unit and $x=h \omega / k_{\mathrm{B}} T$. At higher temperatures the specific heat can take values over the Dulong-Petit limit; therefore, a small anharmonic correction coefficient $\alpha_{\mathrm{D}}$, has been included in Eq. (1). The obtained values of the parameters are: the electronic specific heat coefficient $\gamma=$ $85 \mathrm{~mJ} /\left(\mathrm{mol} \mathrm{K}^{2}\right)$ and the Debye temperature $\Theta_{\mathrm{D}}=325 \mathrm{~K}$, $\alpha_{\mathrm{D}}=2 \times 10^{-4} \mathrm{~K}^{-1}$. The obtained large $\gamma$ value is not a sign of a heavy fermion-type behavior but means only that there are other contributions to the heat capacity in this temperature range, like: spin fluctuations near the ordering temperature $T_{\mathrm{C}}=8 \mathrm{~K}$, crystal field, disorder, etc. In our previous studies on $\mathrm{CeNi}_{4} \mathrm{Si}$ [4] we have not observed any magnetic order down to the lowest temperatures.

The total low-temperature specific heat of a metallic ferromagnet may be written as

$$
C_{p}(T)=\gamma T+\beta T^{3}+\delta T^{3 / 2},
$$

where $\delta T^{3 / 2}$ corresponds to the magnetic correlations [7]. The $\gamma=394 \mathrm{~mJ} /\left(\mathrm{mol} \mathrm{K}{ }^{2}\right)$ parameter was obtained in the temperature range $2-8 \mathrm{~K}$ (inset of Fig. 2). Figure 3 shows the low-temperature part (up to $20 \mathrm{~K}$ ) of the heat capacity of the $\mathrm{NdNi}_{4} \mathrm{Si}$ in the applied magnetic fields to $3 \mathrm{~T}$. Zero field heat capacity of the $\mathrm{NdNi}_{4} \mathrm{Si}$ compound reveals a peak close to the magnetic ordering temperature. This is typical of ferromagnets because larger fields cause a strong magnetization already well above the zero field $T_{\mathrm{C}}$ and the magnetization does not develop spontaneously below a critical temperature anymore. The maximum is shifting to higher temperatures with the increasing magnetic fields.

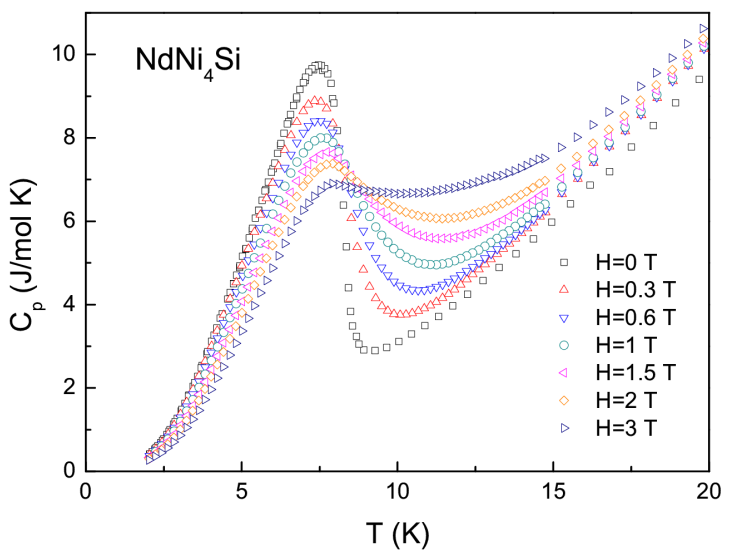

Fig. 3. Specific heat vs. temperature $(2-20 \mathrm{~K})$ for $\mathrm{NdNi}_{4} \mathrm{Si}$ in different applied magnetic fields.

The magnetic contribution to the specific heat and the corresponding magnetic entropy are related to the energy levels of the magnetic ions ( $\mathrm{Nd}$ in our case). The magnetic contribution $C_{\mathrm{m}}$ to the specific heat was calculated as the difference between the measured specific heat and the $C_{\mathrm{el}+\mathrm{ph}}$ contribution obtained from Eq. (1).

The magnetic part of the specific heat is shown in Fig. 4. The maximum above $T_{\mathrm{C}}$ (inset of Fig. 4) can be analyzed in the terms of the Schottky anomaly being a result of the crystal field splitting of the ground state level. The formula for the Schottky contribution is as follows [7]:

$$
\begin{gathered}
C_{\mathrm{Sch}}(T)=\frac{R}{T^{2}}\left[\frac{\sum_{i=0}^{n-1} \Delta_{i}^{2} \mathrm{e}^{-\Delta_{i} / T}}{\sum_{i=0}^{n-1} \mathrm{e}^{-\Delta_{i} / T}}\right. \\
\left.-\left(\frac{\sum_{i=0}^{n-1} \Delta_{i} \mathrm{e}^{-\Delta_{i} / T}}{\sum_{i=0}^{n-1} \mathrm{e}^{-\Delta_{i} / T}}\right)^{2}\right],
\end{gathered}
$$


where $n$ denotes the number of the energy levels and $\Delta_{0}=0$. In the hexagonal symmetry the crystal field splits the $\mathrm{Nd}^{3+}(J=9 / 2)$ multiplet into five Kramers doublets $(n=10)$. The fit presented in Fig. 4 provides the estimation of the energy gaps $\Delta_{1}=62 \mathrm{~K}, \Delta_{2}=85 \mathrm{~K}$.

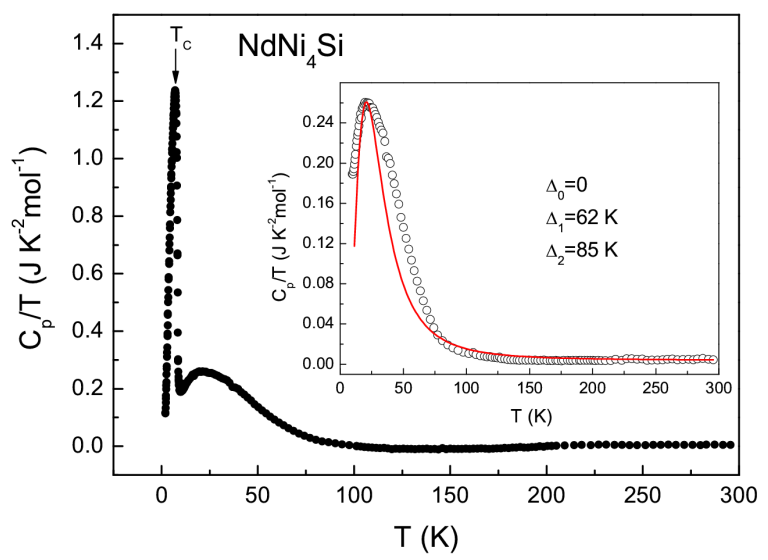

Fig. 4. The magnetic contribution to the specific heat obtained by subtracting the phonon and electronic contribution. Inset: Schottky anomaly above $T_{\mathrm{C}}$ (circles) fitted with Eq. (4) (solid line).

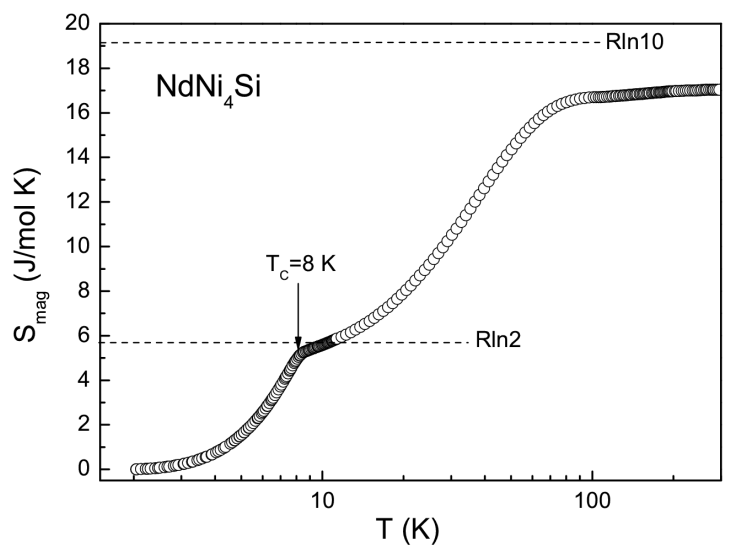

Fig. 5. Temperature dependence of the magnetic entropy on a $\log -T$ scale.

The integration of the area under the peak displayed in Fig. 5 provides the temperature dependence of the magnetic entropy according to the relation

$$
S_{\mathrm{mag}}=\int_{0}^{T} \frac{C_{\mathrm{m}}(T)}{T} \mathrm{~d} T .
$$

The magnetic entropy is related to the degrees of freedom using the expression $S_{\text {mag }}=R \ln (2 J+1)$.
At the ferromagnetic transition point $S_{\text {mag }}$ reaches 100 percent of the value $R \ln 2$ expected for the quasidoublet ground state. At the room temperature the entropy reaches nearly 85 percent of $R \ln 10$.

\section{Conclusions}

The $\mathrm{NdNi}_{4} \mathrm{Si}$ compound undergoes a paraferromagnetic transition at $T_{\mathrm{C}}=8 \mathrm{~K}$ and has a magnetic moment of $1.5 \mu_{\mathrm{B}} /$ f.u. at $4.2 \mathrm{~K}$ (in $H=9 \mathrm{~T})$. The ferromagnetic $\mathrm{NdNi}_{4} \mathrm{Si}$ was characterized by the electronic heat capacity coefficient $\gamma=$ $85 \mathrm{~mJ} /\left(\mathrm{mol} \mathrm{K}^{2}\right)$ and the Debye temperature $\Theta_{\mathrm{D}}=325 \mathrm{~K}$. Zero field heat capacity reveals a peak close to the magnetic ordering temperature. The maximum is shifting to higher temperatures with increasing magnetic fields. The analysis of the Schottky peak appearing in the magnetic part of the specific heat has provided the scheme of the energy levels being a result of the splitting by the crystal electric field. For $\mathrm{Nd}$, three Kramers doublets with $\Delta_{0}=0, \Delta_{1}=62 \mathrm{~K}, \Delta_{2}=85 \mathrm{~K}$ have been obtained.

\section{Acknowledgments}

This work was supported by the funds for science in years 2007-2009 as a research project (A. Kowalczyk, M. Falkowski) and partly by Science and Technology Assistance Agency - APVT-51-031704 by VEGA 6165 by the contract $\mathrm{CE}$ of SAS.

\section{References}

[1] M. Falkowski, B. Andrzejewski, A. Kowalczyk, J. Alloys Comp. 442, 155 (2007).

[2] W.E. Wallace, Rare Earth Intermetallics, Ed. A.M. Alper, J.L. Margrave, A.S. Nowick, Academic Press, New York 1973, p. 266.

[3] J.A. Blanco, D. Gignoux, D. Schmitt, A. Tari, F.Y. Zhang, J. Phys., Condens. Matter 6, 4335 (1994).

[4] A. Kowalczyk, M. Falkowski, V.H. Tran, M. Pugaczowa-Michalska, J. Alloys Comp. 440, 13 (2007).

[5] T. Toliński, W. Schäfer, W. Kockelmann, A. Kowalczyk, A. Hoser, Phys. Rev. B 68, 144403 (2003).

[6] T. Toliński, W. Schäfer, A. Kowalczyk, B. Andrzejewski, A. Hoser, A. Szlaferek, J. Alloys Comp. 385 , 28 (2004).

[7] A. Tari, The Specific Heat of Master at Low Temperatures, Imperial College Press, London 2003, p. 150. 